\title{
Workflow documentation
}

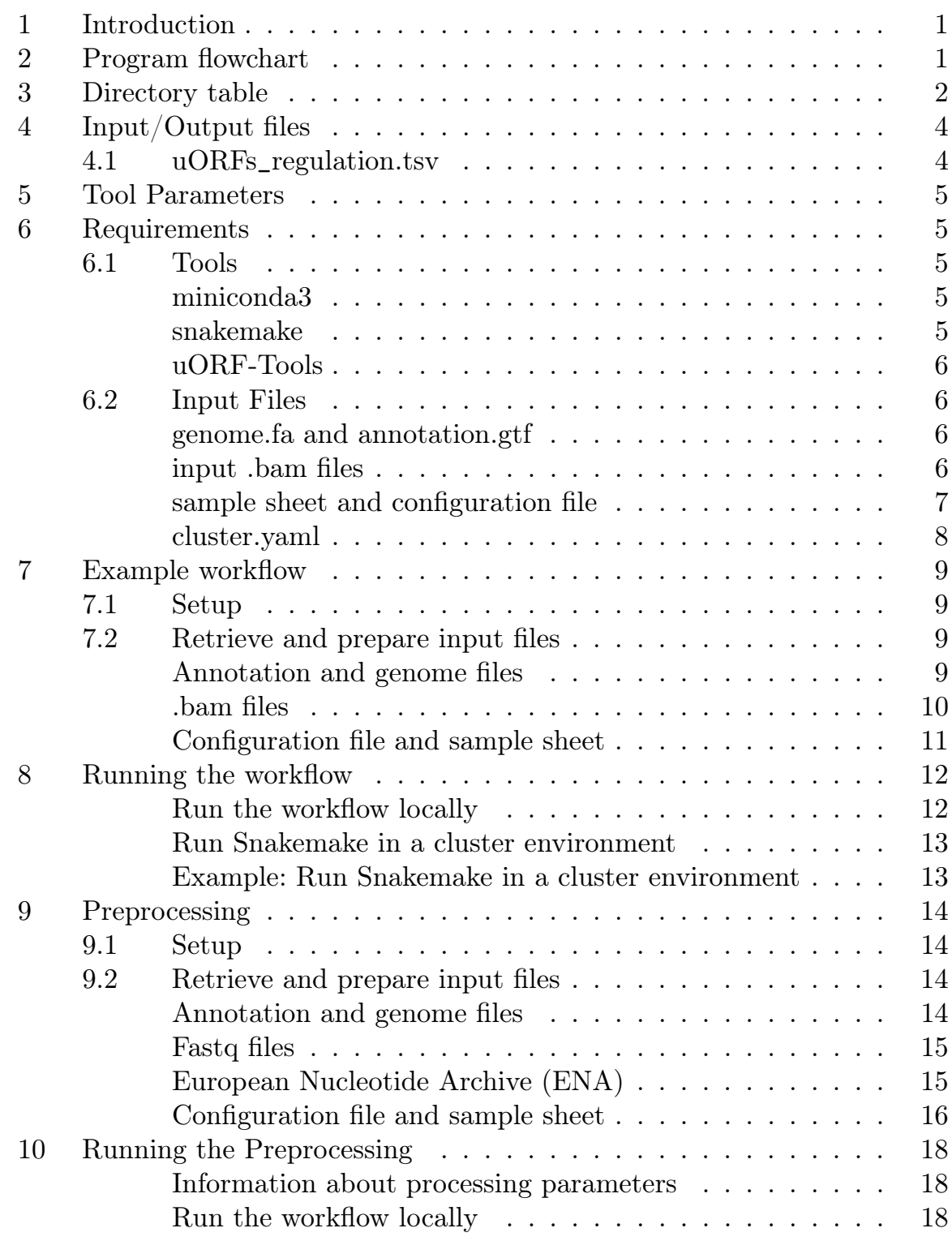


Run Snakemake in a cluster environment . . . . . . . . . 18

Example: Run Snakemake in a cluster environment. . . . 19 


\section{Introduction}

uORF-Tools is a workflow and a collection of tools for the analysis of 'Upstream Open Reading Frames' (short uORFs). The workflow is based on the workflow management system snakemake [1] and handles installation of all dependencies via bioconda [2], as well as all processings steps. The source code of $u O R F$-Tools is open source and available under the License GNU General Public License 3. Installation and basic usage is described below.

This documentation was last updated 08.03.2019. An up-to-date webbased version of the documentation can be found on ReadTheDocs.

\section{Program flowchart}

The flowchart (see Figure 1) describes the processing steps of the workflow and how they are connected.

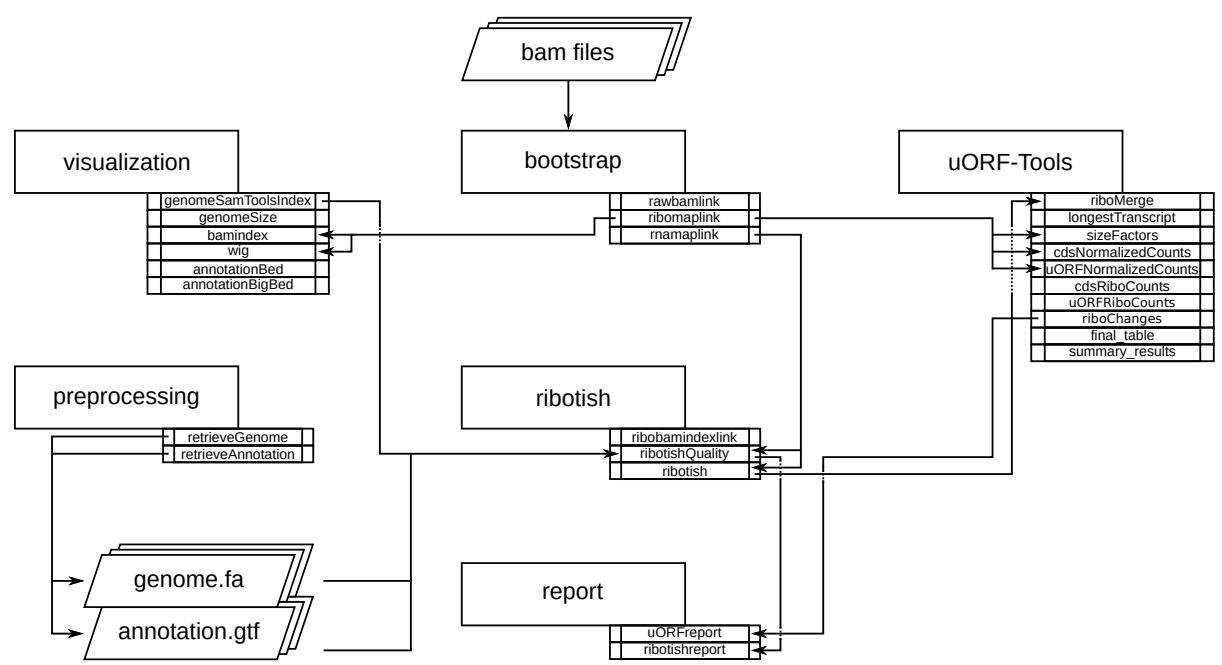

Fig. 1. Sketch of the workflow. 


\section{Directory table}

The output is written to a directory structure (see Figure 2 that corresponds to the workflow steps.

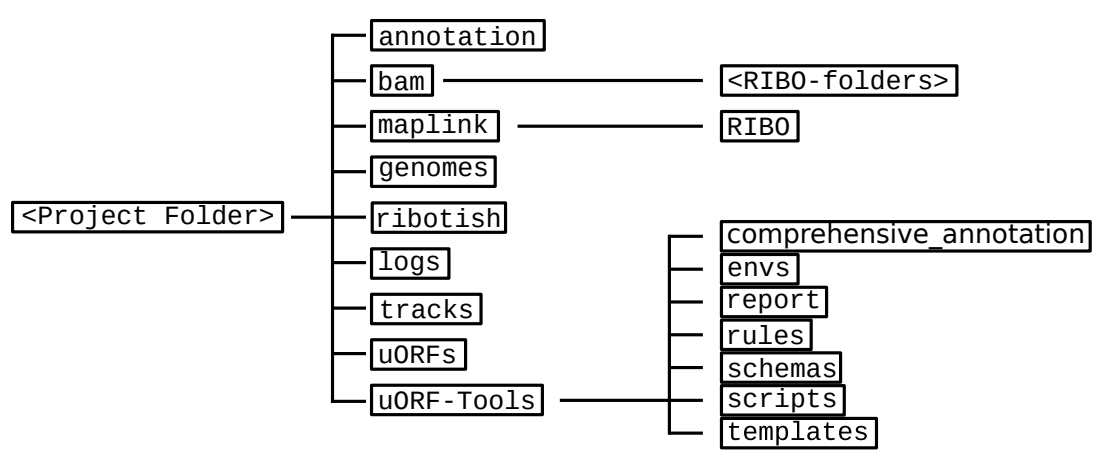

Fig. 2. The directory structure created by running the workflow.

- annotation: contains the processed user-provided annotation file with genomic features.

Contents: annotation.gtf

- bam: contains the input .bam files.

Contents: <method-condition-replicate>.bam

- genomes: contains the genome file, as well as an according index and sizes file.

Contents: genome.fa, genome.fa.fai, sizes.genome

- index: contains an index for the rRNA databases created using indexdb_rna. Contents: $<$ database-ID>.bursttrie_0.dat, $<$ database-ID>.kmer_0.dat,

$<$ database-ID>.pos_0.dat, <database-ID>.stats

- logs: contains log files for each step of the workflow.

Contents: $<$ rule $>. o<j o b I D>,<$ methods $>$.log

- maplink: contains soft links to the .bam files and an according index.

- RIBO: contains soft links to the .bam and .bam.bai files for RIBO and corresponding parameter files (.para.py).

Contents: <method-condition-replicate $>$.bam.bai,

$R I B O /<$ condition-replicate $>$.bam.para.py

- ribotish: contains the result files of ribotish.

Contents: <condition-replicate>-newORFs.tsv,

$<$ condition-replicate $>-n e w O R F$ s.tsv_all.txt,

$<$ condition-replicate $>$-qual.txt, <condition-replicate>-qual.pdf 
- tracks: contains $B E D$ (.bed), wig (.wig) and bigWig (.bw) files for visualizing tracks in a genome browser.

Contents: annotation.bb, annotation.bed, annotation.bed6, annotationNScore.bed6, annotation-woGenes.gtf, $<$ method-condition-replicate $>$.bw $<$ method-condition-replicate $>$.wig

- uORFs: contains the main output of the workflow.

- uORFs_regulation.tsv: table summarizing the predicted uORFs with their regulation on the main ORF.

- merged_uORFs.bed: genome browser track with predicted uORFs.

Contents: longest_protein_coding_transcripts.gtf, uORF_regulation.tsv, ribo_norm_CDS_reads.csv, ribo_norm_uORFs_reads.csv, ribo_raw_CDS_reads.csv, ribo_raw_uORFs_reads.csv, sfactors_lprot.csv, merged_uORFs.bed, merged_uORFs.csv,

- uORF-Tools: contains the workflow tools.

- comprehensive_annotation: an example annotation.

- envs: conda environment files (.yaml).

- report: restructuredText files for the report (.rst).

- rules: the snakemake rules.

- schemas: validation templates for input files

- scripts: scripts used by the snakemake workflow.

- templates: templates for the config.yaml and the samples.tsv. 


\section{Input/Output files}

The following table contains explanations for each of the input/output files:

\begin{tabular}{|c|c|}
\hline File name & Description \\
\hline annotation.gtf & user-provided annotation file with genomic features \\
\hline genome.fa & user-provided genome file containing the genome sequence \\
\hline genome.fa.fai & index file of the genome file \\
\hline sizes.genome & file containing the sizes of each genome sequence in the genome file \\
\hline$<$ method $>-<$ conditon $>-<$ replicate $>$.bam & user-provided alignment files (or created using the preprocessing workflow \\
\hline$<$ method-condition-replicate $>$.bam.bai & index file of the alignment files \\
\hline$<$ condition-replicate $>$.bam.para.py & parameter file generated by RiboTISH \\
\hline$<$ methods $>\log$ & files containing the process $\log$ for each method \\
\hline$<$ condition-replicate $>$-newORFs.tsv & RiboTISH output file containing newly discovered ORFs (significant only) \\
\hline$\langle$ <ondition-replicate $>$-newORFs.tsv_all.txt & RiboTISH output file containing newly discovered ORFs (all) \\
\hline$<$ condition-replicate $>$-qual.txt & RiboTISH quality control report text file \\
\hline$<$ condition-replicate $>$-qual.pdf & RiboTISH quality control report file with illustrations \\
\hline annotation.bb & input annotation in bigbed format for genome browser visualization \\
\hline annotation.bed & input annotation in bed format for genome browser visualization \\
\hline annotation.bed 6 & input annotation in bed6 format for genome browser visualization \\
\hline annotation-woGenes.gtf & input annotation filtered exclusively for gene features \\
\hline$<$ method-condition-replicate $>$.bw & BigWig files for visualizing data in a genome Browser \\
\hline$<$ method-condition-replicate $>$.wig & wig files for visualizing data in a genome Browser \\
\hline uORFs_regulation.tsv & final output table including all $\mathrm{uORFs}$ and their $\mathrm{mORF}$ \\
\hline merged_uORFs.bed & bed file containing potential ORFs for genome browser visualization \\
\hline merged_uORFs.csv & list of potential ORFs with coordinates and mORF annotation \\
\hline longest_protein_coding_transcripts.gtf & input annotation filtered for longest splice variant for each locus \\
\hline ribo_raw_CDS_reads.csv & read counts for annotated ORFs \\
\hline ribo_raw_uORFs_reads.csv & read counts for potential ORFs \\
\hline ribo_norm_CDS_reads.csv & deseq2 normalized read counts for annotated ORFs \\
\hline ribo_norm_uORFs_reads.csv & deseq2 normalized read counts for potential ORFs \\
\hline sfactors_lprot.csv & deseq2 size factors for protein coding transcripts \\
\hline
\end{tabular}

\section{1 uORFs_regulation.tsv}

Description for the columns present in the final output file (Supplement Table 3):

- transcript_id: transcript id of the main open reading frame (mORF)

- uORF_id: id of the potential Upstream open reading frame (uORF), derived from mORF id

- Ratio: list of columns, one for each sample, with the ratio of read counts for the mORF and the uORF

- Standard deviation of changes of the ratio of the relative uORF activities of treatment vs control

- binary logarithm fold change of the ratio of the relative $\mathrm{uORF}$ activities of treatment vs control 


\section{Tool Parameters}

Special characters and versions used for the most important tools. Standard input/output parameters were omitted.

\begin{tabular}{|l|l|l|}
\hline Tool & Version & Special parameters used \\
\hline riboTISH & 0.2 .1 & -longest (-v -p -b -g -f) \\
\hline trim-galore & 0.5 .0 & -phred33 -q 20 -length 15 -trim-n -suppress_warn -clip_R1 1 -dont_gzip \\
\hline star & $2.6 .1 b$ & $\begin{array}{l}\text {-genomeDir genomeStarIndex -outSAMtype BAM SortedByCoordinate } \\
\text {-outSAMattributes All -outFilterMultimapNmax 1 -alignEndsType Extend5pOfRead1 }\end{array}$ \\
\hline sortmerna & $2.1 b$ & -m 4096 -a -ref < dbstring > -reads -num_alignments 1 -fastx -aligned -other \\
\hline fastqc & 0.11 .8 & \\
\hline imagemagick & $7.0 .8 \_15$ & -density 150 -trim -quality 100 -flatten -sharpen 0x1.0 \\
\hline
\end{tabular}

\section{Requirements}

In the following, we describe all the required files and tools needed to run our workflow.

\subsection{Tools}

\section{miniconda3}

As this workflow is based on the workflow management system snakemake [1]. Snakemake will download and install all necessary dependencies via conda. We strongly recommend installing miniconda3 with python3.7.

After downloading the miniconda3 version suiting your linux system, execute the downloaded bash file and follow the instructions given.

\section{snakemake}

The uORF-Tools require snakemake (version $==\mathbf{5 . 4 . 5}$ ).

The newest version of snakemake can be downloaded via conda using the following command:

$$
\begin{aligned}
\$ \text { conda create } & -c \text { conda-forge -c bioconda } \backslash \\
& -n \text { snakemake snakemake }==5.4 .5
\end{aligned}
$$

This creates a new conda environment called snakemake and installs snakemake into the environment.

The environment can be activated using:

$$
\text { \$ conda activate snakemake }
$$

and deactivated using:

\$ conda deactivate 


\section{uORF-Tools}

Using the workflow requires the $u O R F$-Tools. The latest version is available on our GitHub page.

In order to run the workflow, we suggest that you download the $u O R F$-Tools into your project directory. The following command creates an example directory and changes into it:

\$ mkdir project; cd project;

Now, download and unpack the latest version of the $u O R F$-Tools by entering the following commands:

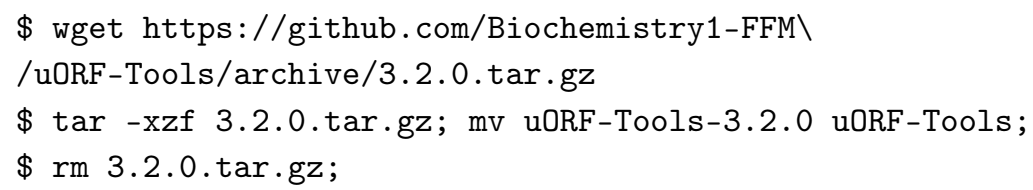

The $u O R F$-Tools are now located in a subdirectory of your workflow directory.

\subsection{Input Files}

Several input files are required in order to run our workflow, a genome sequence (.fa), an annotation file (.gtf) and the bam files (.bam).

\section{genome.fa and annotation.gtf}

We recommend retrieving both the genome and the annotation files for mouse and human from GENCODE 3] and for other species from Ensembl Genomes [4.

\section{input .bam files}

These are the input files provided by you (the user).

"For best performance, reads should be trimmed (to 29 nt RPF length) and aligned to genome using end-to-end mode (no soft-clip). Intron splicing is supported. Some attributes are needed such as NM, NH and MD. For STAR, 'outSAMattributes All' should be set. bam file should be sorted and indexed by samtools." (RiboTISH requirements, see https://github.com/zhpn1024/ribotish ) .

Please ensure that you move all input .bam files into a folder called bam:

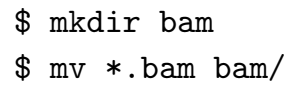




\section{sample sheet and configuration file}

In order to run the $u O R F$-Tools, you have to provide a sample sheet and a configuration file. There are templates for both files available in the $u O R F$-Tools folder.

Copy the templates of the sample sheet and the configuration file into the $u O R F$ Tools folder:

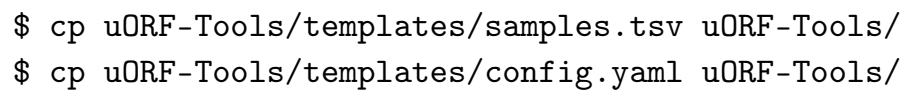

Customize the config.yaml using your preferred editor. It contains the following variables:

- taxonomy Specify the taxonomic group of the used organism in order to ensure the correct removal of reads mapping to ribosomal genes (Eukarya, Bacteria, Archea). (Option for the preprocessing workflow)

- adapter Specify the adapter sequence to be used. If not set, Trim galore will try to determine it automatically. (Option for the preprocessing workflow)

- samples The location of the samples sheet created in the previous step.

- genomeindexpath If the STAR genome index was already precomputed, you can specify the path to the files here, in order to avoid recomputation. (Option for the extended workflow)

- uorfannotationpath If a uORF-annotation file was already pre-computed, you can specify the path to the file here. Please make sure, that the file has the same format as the uORF_annotation_hg38.csv file provided in the git repo (i.e. same number of columns, same column names)

- alternativestartcodons Specify a comma separated list of alternative start codons.

Edit the sample sheet corresponding to your project. It contains the following variables:

- method Indicates the method used for this project, here RIBO for ribosome profiling.

- condition Indicates the applied condition (A, B)

- replicate Used to distinguish between the different replicates (e.g. $1,2, \ldots$ )

- inputFile Indicates the bam file for a given sample.

As seen in the samples.tsv template:

Please make sure that you have at-least two replicates for each condition!.

Please ensure that you put the treatment before the control alphabetically (e.g. A: Treatment B: Control). 


\begin{tabular}{|l|l|l|l|}
\hline method & condition & replicate & inputFile \\
\hline RIBO & A & 1 & bam/RIBO-A-1.bam \\
\hline RIBO & A & 2 & bam/RIBO-A-2.bam \\
\hline RIBO & A & 3 & bam/RIBO-A-3.bam \\
\hline RIBO & A & 4 & bam/RIBO-A-4.bam \\
\hline RIBO & B & 1 & bam/RIBO-B-1.bam \\
\hline RIBO & B & 2 & bam/RIBO-B-2.bam \\
\hline RIBO & B & 3 & bam/RIBO-B-3.bam \\
\hline RIBO & B & 4 & bam/RIBO-B-4.bam \\
\hline
\end{tabular}

\section{cluster.yaml}

In the template folder, we provide two cluster.yaml files needed by snakemake in order to run on a cluster system:

- sge-cluster.yaml for grid based queuing systems

- torque-cluster.yaml for torque based queuing systems 


\section{Example workflow}

The retrieval of input files and running the workflow locally and on a server cluster via a queuing system is demonstrated using an example with data available from our FTP-Server.

Ensure that you have miniconda3 installed and a conda environment set-up. Please refer to the Tools Section (6.1) for details on the installation.

\subsection{Setup}

First of all, we start by creating the project directory and changing to it.

$\$$ mkdir project; cd project;

We then download the latest version of the $u O R F$-Tools into the newly created project folder and unpack it.

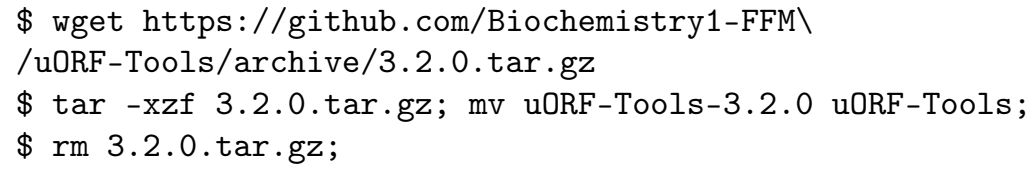

\section{2 $\quad$ Retrieve and prepare input files}

Before starting the workflow, we have to acquire and prepare several input files. These files are the annotation file, the genome file, the bam files, the configuration file and the sample sheet.

\section{Annotation and genome files}

First, we want to retrieve the annotation file and the genome file. In this case, we can find both on the GENCODE [3] webpage for the human genome.

On this page, we can directly retrieve both files by clicking on the according download links next to the file descriptions. (As shown in Figure 3). Alternatively, you can directly download them using the following commands:

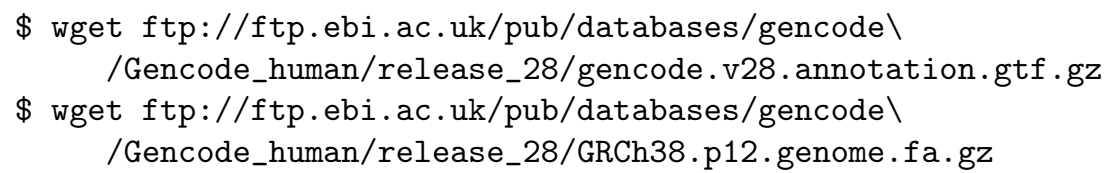




\begin{tabular}{|c|c|c|c|}
\hline Content & Regions & Description & Download \\
\hline $\begin{array}{l}\text { Comprehensive gene } \\
\text { annotation }\end{array}$ & $\mathrm{CHR}$ & $\begin{array}{l}\text { - It contains the comprehensive gene annotation on the reference chromosomes only } \\
\text { - This is the main annotation file for most users }\end{array}$ & $\rightarrow$ GTF \\
\hline $\begin{array}{l}\text { Comprehensive gene } \\
\text { annotation }\end{array}$ & ALL & $\begin{array}{l}\text { - It contains the comprehensivivg gene annotation on the reference chromosomes, scaffolds, assembly } \\
\text { patches and ditemate loci (haplotypes) } \\
\text { - This is a superset of the main annotation file }\end{array}$ & $\mathrm{GTF} \oplus \mathrm{GFF} 3$ \\
\hline \multicolumn{4}{|l|}{ Fasta files } \\
\hline Content & Regions & Description & Download \\
\hline Transcript sequences & CHR & - Nucleotide sequences of all transcripts on the reference chromosomes & Fasta \\
\hline $\begin{array}{l}\text { Protein-coding transcript } \\
\text { sequences }\end{array}$ & $\mathrm{CHR}$ & 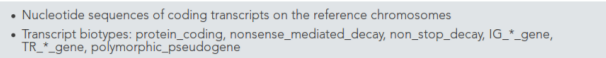 & Fasta 9 \\
\hline $\begin{array}{l}\text { Protein-coding transcript } \\
\text { translation sequences }\end{array}$ & $\mathrm{CHR}$ & 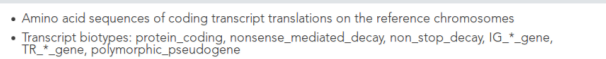 & Fasta \\
\hline $\begin{array}{l}\text { Long non-coding RNA } \\
\text { transcript sequences }\end{array}$ & CHR & - Nucleotide sequences of long non-coding RNA transcripts on the reference chromosomes & Fasta 1 \\
\hline $\begin{array}{l}\text { Genome sequence } \\
\text { (GRCh38.p12) }\end{array}$ & ALL & $\begin{array}{l}\text { - Nucleotide sequence of the GRCh38,p12 genome assembly version on all regions, including reference } \\
\text { chromosomess, scaffolds, assembly patches and haplotypes } \\
\text { - The sequence region names are the same as in the GTF/GFF files }\end{array}$ & Fasta \\
\hline
\end{tabular}

Fig. 3. Downloading the annotation file and the genome file from the GenCode webpage using the direct download links.

Then, we are going to unpack both files.

\$ gunzip gencode.v28.annotation.gtf.gz

\$ gunzip GRCh38.p12.genome.fa.gz

Finally, we will rename these files to annotation.gtf and genome.fa.

\$ mv gencode.v28.annotation.gtf annotation.gtf

$\$$ mv GRCh38.p12.genome.fa genome.fa

Another webpage that provides these files is Ensembl Genomes. This usually requires searching their file system in order to find the wanted files. For this tutorial, we recommend to stick to GenCode instead.

\section{.bam files}

Next, we want to acquire the bam files. The bam files for the tutorial dataset can be downloaded from our FTP-Server:

We provide both a .zip and a .tar.gz file. We recommend the .tar.gz file as most linux systems can decompress them via commandline by default.

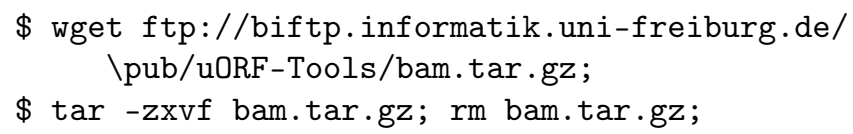

This will create a bam folder containing all the files necessary to run the workflow. If you prefer using your own .bam files, we suggest creating a bam folder and copying the files into it. Make sure that your reads were trimmed (to $\sim 29 \mathrm{bp}$ length) and aligned to the genome using end-to-end alignment. The bam files need to include all SAM attributes and should be sorted using samtools. 
\$ mkdir bam; mv *.bam bam/;

\section{Configuration file and sample sheet}

Finally, we will prepare the configuration file (config.yaml) and the sample sheet (samples.tsv). We start by copying templates for both files from the $u O R F$ Tools/templates into the uORF-Tools/ folder.

\$ cp uORF-Tools/templates/bam-samples.tsv uORF-Tools/

$\$ \mathrm{mv}$ uORF-Tools/bam-samples.tsv uORF-Tools/samples.tsv

The standard template bam-samples.tsv looks as follows:

\begin{tabular}{|l|l|l|l|}
\hline method & condition & replicate & inputFile \\
\hline RIBO & A & 1 & bam/RIBO-A-1.bam \\
\hline RIBO & A & 2 & bam/RIBO-A-2.bam \\
\hline RIBO & A & 3 & bam/RIBO-A-3.bam \\
\hline RIBO & A & 4 & bam/RIBO-A-4.bam \\
\hline RIBO & B & 1 & bam/RIBO-B-1.bam \\
\hline RIBO & B & 2 & bam/RIBO-B-2.bam \\
\hline RIBO & B & 3 & bam/RIBO-B-3.bam \\
\hline RIBO & B & 4 & bam/RIBO-B-4.bam \\
\hline
\end{tabular}

When using your own data, use any editor (vi(m), gedit, nano, atom, ...) to customize the sample sheet.

Please ensure not to replace any tabulator symbols with spaces while changing this file.

Next, we are going to set up the config.yaml.

\$cp uORF-Tools/templates/config.yaml uORF-Tools/

$\$$ vi uORF-Tools/config.yaml

This file contains the following variables:

- taxonomy Specify the taxonomic group of the used organism in order to ensure the correct removal of reads mapping to ribosomal genes (Eukarya, Bacteria, Archea). (Option for the preprocessing workflow)

- adapter Specify the adapter sequence to be used. If not set, Trim galore will try to determine it automatically. (Option for the preprocessing workflow)

- samples The location of the samples sheet created in the previous step.

- genomeindexpath If the STAR genome index was already precomputed, you can specify the path to the files here, in order to avoid recomputation. (Option for the preprocessing workflow) 
- uorfannotationpath If a uORF-annotation file was already pre-computed, you can specify the path to the file here. Please make sure, that the file has the same format as the uORF_annotation_hg38.csv file provided in the git repo (i.e. same number of columns, same column names)

- alternativestartcodons Specify a comma separated list of alternative start codons.

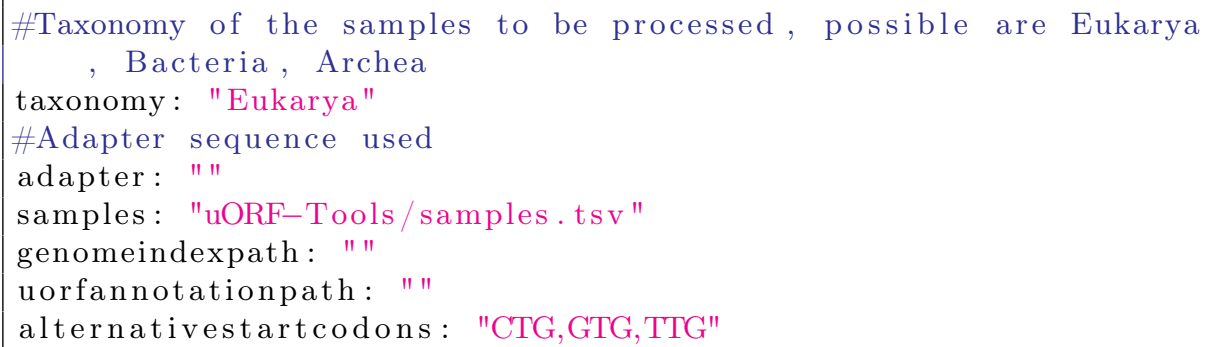

Fig. 4. The contents of the configuration file (config.yaml)

For this tutorial, we can keep the default values for the config.yaml as shown in Figure 6. The organism analyzed in this tutorial is homo sapiens, therefore we keep the taxonomy at Eukarya. The path to samples.tsv is set correctly.

\section{Running the workflow}

Now that we have all the required files, we can start running the workflow, either locally or in a cluster environment.

Run the workflow locally Use the following steps when you plan to execute the workflow on a single server or workstation. Please be aware that some steps of the workflow require a lot of memory, specifically for eukaryotic species.

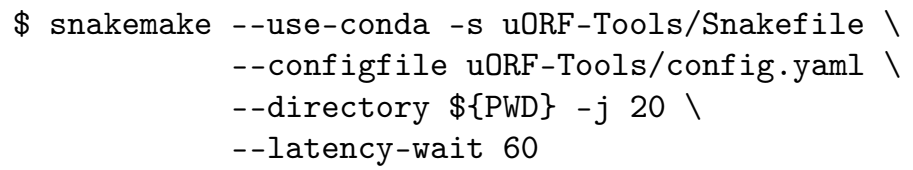




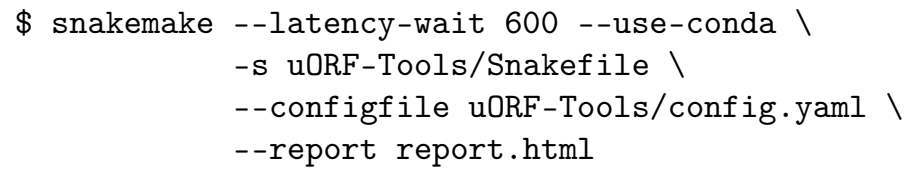

\section{$9 \quad$ Preprocessing}

The retrieval of input files and running the workflow locally and on a server cluster via a queuing system is demonstrated using an example with data available from SRA via NCBI. The dataset is available under the GEO accession number GSE103719. The retrieval of the data is described in this tutorial.

Ensure that you have miniconda3 installed and a conda environment set-up. Please refer to the Tools Section (6.1) for details on the installation.

\subsection{Setup}

First of all, we start by creating the project directory and changing to it.

\$ mkdir preprocessing_project; cd preprocessing_project;

We then download the latest version of the $u O R F$-Tools into the newly created project folder and unpack it.

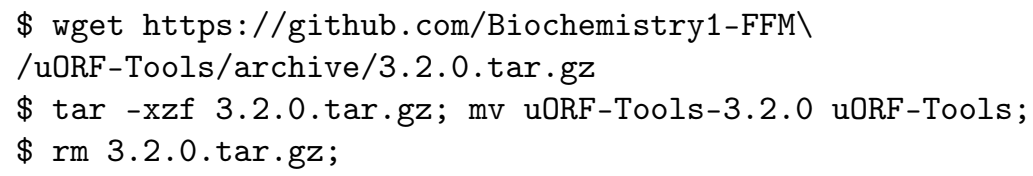

\subsection{Retrieve and prepare input files}

Before starting the workflow, we have to acquire and prepare several input files. These files are the annotation file, the genome file, the fastq files, the configuration file and the sample sheet.

\section{Annotation and genome files}

On this page, we can directly retrieve both files by clicking on the according download links next to the file descriptions. (As shown in Figure 3). Alternatively, you can directly download them using the following commands:

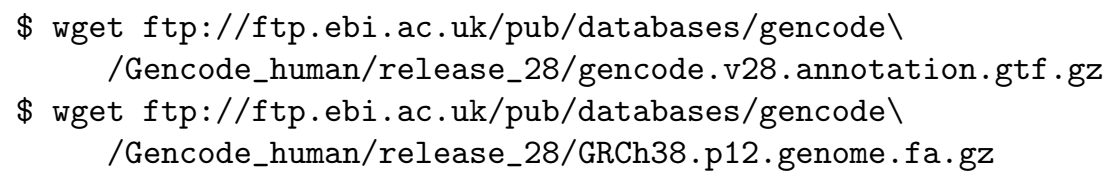


Then, we are going to unpack both files.

$\$$ gunzip gencode.v28.annotation.gtf.gz

\$ gunzip GRCh38.p12.genome.fa.gz

Finally, we will rename these files to annotation.gtf and genome.fa.

$\$ \mathrm{mv}$ gencode.v28.annotation.gtf annotation.gtf

$\$$ mv GRCh38.p12.genome.fa genome.fa

Another webpage that provides these files is Ensembl Genomes. This usually requires searching their file system in order to find the wanted files. For this tutorial, we recommend to stick to GenCode instead.

\section{Fastq files}

In this example, we will use both RNA-seq and RIBO-seq data. In order to fasten up the tutorial, we download only 2 of the 4 replicates available for each Condition.

Please note that you should always use all available replicates, when analyzing your data.

\section{European Nucleotide Archive (ENA)}

For many datasets, the easiest way to retrieve the fastq files is using the European Nucleotide Archive as it provides direct download links when searching for a dataset. Use the interface on ENA or type the follwing commands:

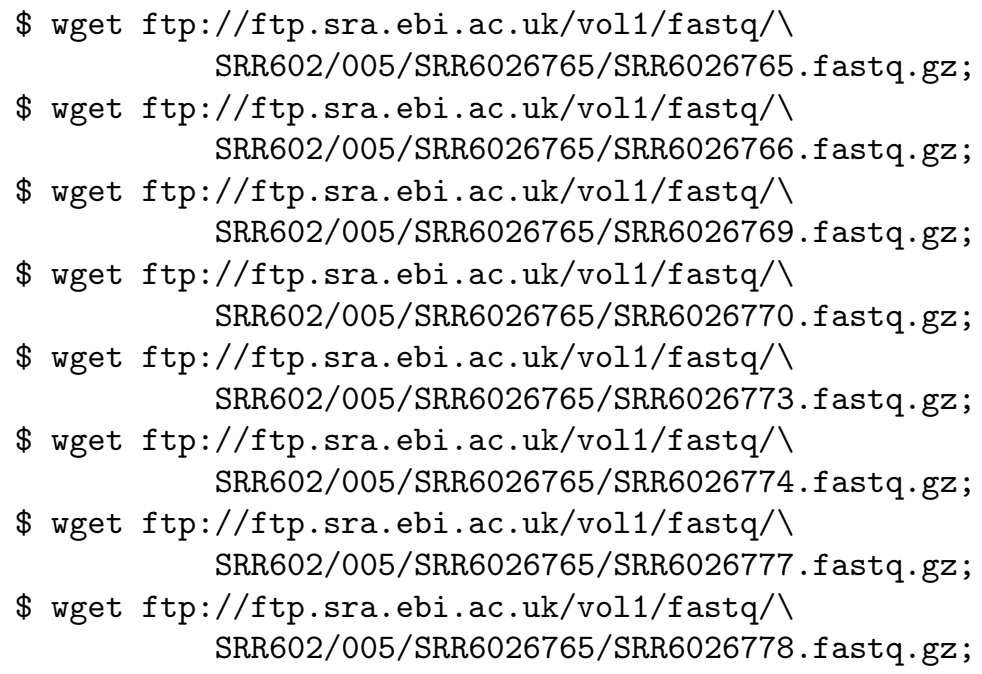

Then, we create a fastq folder and move all the fastq.gz files into this folder.

\$ mkdir fastq; mv *.fastq.gz fastq/; 


\section{Configuration file and sample sheet}

Finally, we will prepare the configuration file (config.yaml) and the sample sheet (samples.tsv). We start by copying templates for both files from the uORFTools/templates into the uORF-Tools/folder.

\$ cp uORF-Tools/templates/fastq-samples.tsv uORF-Tools/

The template looks as follows:

\begin{tabular}{|l|l|l|l|}
\hline method & condition & replicate & inputFile \\
\hline RNA & A & 1 & fastq/SRR6026769.fastq.gz \\
\hline RNA & A & 2 & fastq/SRR6026770.fastq.gz \\
\hline RNA & B & 1 & fastq/SRR6026765.fastq.gz \\
\hline RNA & B & 2 & fastq/SRR6026766.fastq.gz \\
\hline RIBO & A & 1 & fastq/SRR6026777.fastq.gz \\
\hline RIBO & A & 2 & fastq/SRR6026778.fastq.gz \\
\hline RIBO & B & 1 & fastq/SRR6026773.fastq.gz \\
\hline RIBO & B & 2 & fastq/SRR6026774.fastq.gz \\
\hline
\end{tabular}

Please ensure not to replace any tabulator symbols with spaces while changing this file.

Next, we are going to set up the config.yaml.

\$ cp uORF-Tools/templates/config.yaml uORF-Tools/

$\$$ vi uORF-Tools/config.yaml

This file contains the following variables:

- taxonomy Specify the taxonomic group of the used organism in order to ensure the correct removal of reads mapping to ribosomal genes (Eukarya, Bacteria, Archea). (Option for the preprocessing workflow)

- adapter Specify the adapter sequence to be used. If not set, Trim galore will try to determine it automatically. (Option for the preprocessing workflow)

- samples The location of the samples sheet created in the previous step.

- genomeindexpath If the STAR genome index was already precomputed, you can specify the path to the files here, in order to avoid recomputation. (Option for the preprocessing workflow)

- uorfannotationpath If a uORF-annotation file was already pre-computed, you can specify the path to the file here. Please make sure, that the file has the same format as the uORF_annotation_hg38.csv file provided in the git repo (i.e. same number of columns, same column names)

- alternativestartcodons Specify a comma separated list of alternative start codons. 
Change the config file as shown in Figure 6. For this tutorial, we can keep the

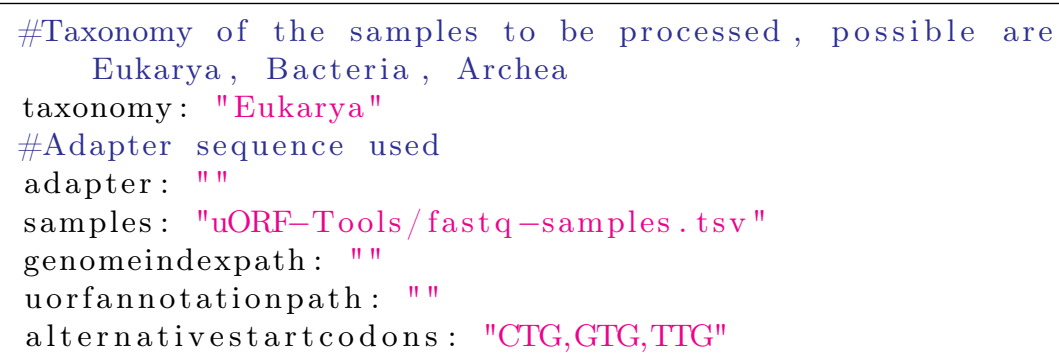

Fig. 6. The contents of the configuration file (config.yaml)

default values for the config.yaml as shown in Figure 6. The organism analyzed in this tutorial is homo sapiens, therefore we keep the taxonomy at Eukarya. The path to samples.tsv is set correctly. 


\section{Running the Preprocessing}

Now that we have all the required files, we can start running the workflow, either locally or in a cluster environment.

Information about processing parameters In this pipeline we use trim_galore for adapter and quality trimming (Parameters: -phred33 -q 20 -length 15 -trim-n -suppress_warn -clip_R1 1), sortmerna for rRNA removal (rRNA fasta files are obtained from https://github.com/biocore/sortmerna/tree/master/rRNA_databases , according to the specified Taxon (i.e. Eukarya, Bacteria, Archea)) and STAR for read alignment.

(Parameters: -outSAMtype BAM SortedByCoordinate -outSAMattributes All -outFilterMultimapNmax 1

-alignEndsType Extend5pOfRead1).

Run the workflow locally Use the following steps when you plan to execute the workflow on a single server or workstation. Please be aware that some steps of the workflow require a lot of memory, specifically for eukaryotic species.

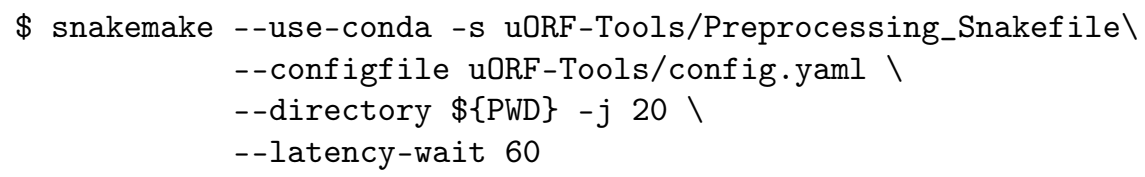

Run Snakemake in a cluster environment Use the following steps if you are executing the workflow via a queuing system. Edit the configuration file cluster.yaml according to your queuing system setup and cluster hardware. The following system call shows the usage with Grid Engine:

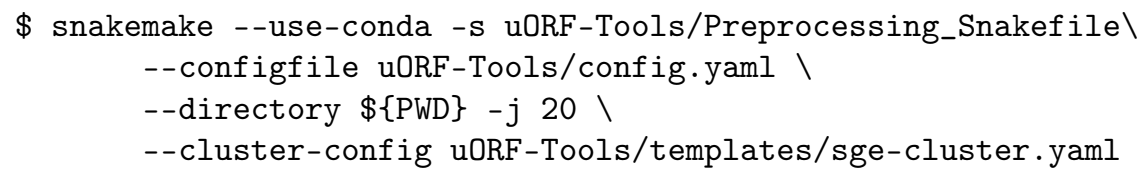




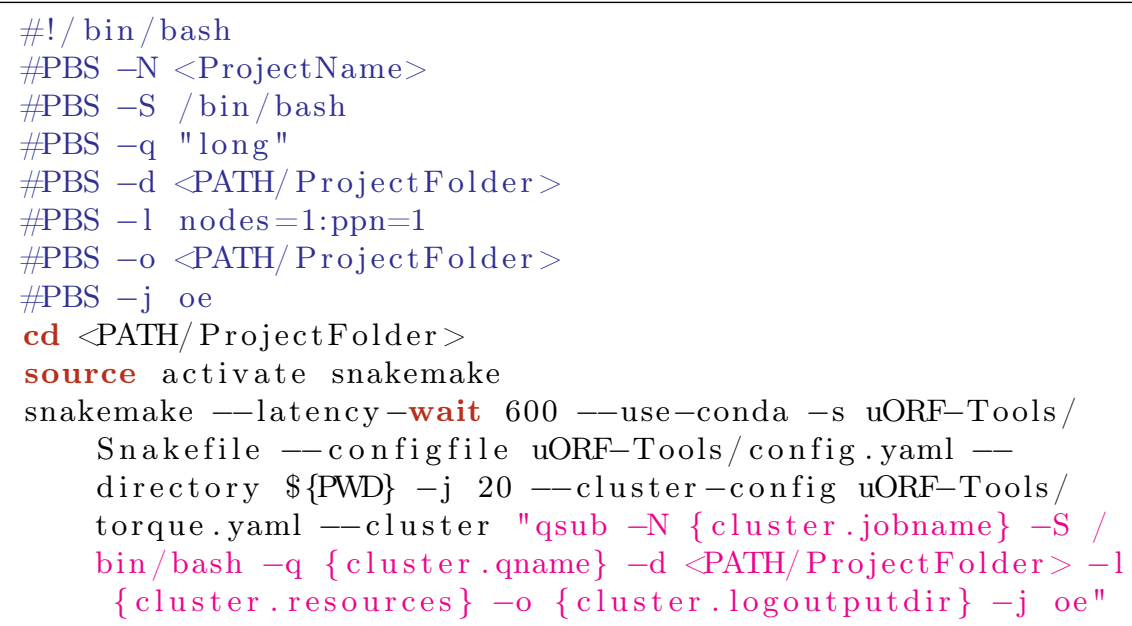

Fig. 7. Bash script to run the example workflow in a TORQUE cluster environment.

Example: Run Snakemake in a cluster environment We ran the tutorial workflow in a cluster environment, specifically a TORQUE cluster environment. Therefore, we created a bash script torque.sh in our project folder.

\$ vi torque.sh

We proceeded by writing the queueing script as shown in Figure 7 We then simply submitted this job to the cluster.

\$ qsub torque.sh

Using any of the presented methods, this will run the workflow on our dataset and create the desired output files. 


\section{Bibliography}

[1] Johannes Köster and Sven Rahmann. SnakemakeâǍTa scalable bioinformatics workflow engine. Bioinformatics, page bty350, 2018.

[2] Björn Grüning, Ryan Dale, Andreas Sjödin, Jillian Rowe, Brad A. Chapman, Christopher H. Tomkins-Tinch, Renan Valieris, and Johannes Köster. Bioconda: A sustainable and comprehensive software distribution for the life sciences. bioRxiv, 2017.

[3] J. Harrow, A. Frankish, J. M. Gonzalez, E. Tapanari, M. Diekhans, F. Kokocinski, B. L. Aken, D. Barrell, A. Zadissa, S. Searle, I. Barnes, A. Bignell, V. Boychenko, T. Hunt, M. Kay, G. Mukherjee, J. Rajan, G. Despacio-Reyes, G. Saunders, C. Steward, R. Harte, M. Lin, C. Howald, A. Tanzer, T. Derrien, J. Chrast, N. Walters, S. Balasubramanian, B. Pei, M. Tress, J. M. Rodriguez, I. Ezkurdia, J. van Baren, M. Brent, D. Haussler, M. Kellis, A. Valencia, A. Reymond, M. Gerstein, R. Guigo, and T. J. Hubbard. GENCODE: the reference human genome annotation for The ENCODE Project. Genome Res., 22(9):1760-1774, Sep 2012.

[4] Daniel R Zerbino, Premanand Achuthan, Wasiu Akanni, MÂăRidwan Amode, Daniel Barrell, Jyothish Bhai, Konstantinos Billis, Carla Cummins, Astrid Gall, Carlos GarcÃna GirÃşn, Laurent Gil, Leo Gordon, Leanne Haggerty, Erin Haskell, Thibaut Hourlier, Osagie G Izuogu, Sophie H Janacek, Thomas Juettemann, Jimmy Kiang To, Matthew R Laird, Ilias Lavidas, Zhicheng Liu, Jane E Loveland, Thomas Maurel, William McLaren, Benjamin Moore, Jonathan Mudge, Daniel N Murphy, Victoria Newman, Michael Nuhn, Denye Ogeh, Chuang Kee Ong, Anne Parker, Mateus Patricio, Harpreet Singh Riat, Helen Schuilenburg, Dan Sheppard, Helen Sparrow, Kieron Taylor, Anja Thormann, Alessandro Vullo, Brandon Walts, Amonida Zadissa, Adam Frankish, Sarah E Hunt, Myrto Kostadima, Nicholas Langridge, Fergal J Martin, Matthieu Muffato, Emily Perry, Magali Ruffier, Dan M Staines, Stephen J Trevanion, Bronwen L Aken, Fiona Cunningham, Andrew Yates, and Paul Flicek. Ensembl 2018. Nucleic Acids Research, 46(D1):D754-D761, 2018. 